\title{
Integrated Histogenetic Analysis Reveals BAP1-Mutated Epithelioid Mesothelioma in a Patient With Cancer of Unknown Primary
}

\author{
Tilmann Bochtler, MD,a,b,; Volker Endris, PhD c,; Anna Reiling ; Jonas Leichsenring, MDc; \\ Michal R. Schweiger, MD, PhD,e; Sebastian Klein, MD,e; Fabian Stögbauer, MD; ; Benjamin Goeppert, MDc; \\ Peter Schirmacher, MD ${ }^{c, f} ;$ Alwin Krämer, $\mathrm{MD}^{\mathrm{a}, \mathrm{b}, \uparrow}$; and Albrecht Stenzinger, MD ${ }^{\mathrm{c}, \mathrm{f}, \uparrow}$
}

\begin{abstract}
This case report presents a male patient with epithelioid mesothelioma that was initially misdiagnosed as cancer of unknown primary (CUP) and correctly identified using molecular panel sequencing. The patient had a prior history of colon and breast cancer. To assess the enlarged mediastinal lymph nodes, retrosternal lymphadenectomy was performed in 2016. The lymph nodes were histologically deemed unrelated to the known breast cancer by the reference pathologist, thus leading to the diagnosis of a CUP syndrome. When the patient presented to our center, targeted deep sequencing of both breast cancer and presumed CUP was performed to address the clonal relationship between both malignancies. A missense mutation in BAP1 was revealed in both samples, with coverage data indicating a germline event. The patient was subsequently counseled by a human geneticist and underwent genetic testing, which confirmed the germline nature of this mutation. Collectively, these data led to the diagnosis of BAP1 (BRCA1-associated protein-1) tumor predisposition syndrome (TPDS). With the knowledge of an underlying BAP1 mutation and its known frequent association with epithelioid mesothelioma, the histology was reassessed and the diagnosis was revised to epithelioid mesothelioma. At this point, peritoneal involvement of mesothelioma could be diagnosed and histologically confirmed. This case illustrates the potential of integrated histopathologic and molecular diagnostics in helping to decipher CUP syndromes and establish the correct diagnosis. Additionally, this case highlights typical features of BAP1 TPDS with its general susceptibility to cancers, with pleural and peritoneal mesotheliomas as most prevalent clinical entities and the typically more benign course of these epithelioid mesotheliomas compared with BAP1-unrelated cases of mesotheliomas.
\end{abstract}

Cancer of unknown primary (CUP) is an enigmatic entity in which malignancy is histologically proven by a metastasis specimen, but the primary tumor remains elusive despite extensive clinical workup, including clinical examination; CT of the neck, chest, and abdomen; gastroscopy and colonoscopy; comprehensive laboratory

\footnotetext{
${ }^{a}$ Clinical Cooperation Unit Molecular Hematology/Oncology, German Cancer Research Center (DKFZ), Heidelberg; 'bepartment of Internal Medicine V, University Hospital Heidelberg, Heidelberg; 'Institute of Pathology, University Hospital Heidelberg, Heidelberg; 'Institute of Human Genetics, University Hospital Cologne, Cologne; éaboratory for Translational Epigenetics and Tumor Genetics, Cologne; and ${ }^{\mathrm{f} G e r m a n}$ Cancer Consortium (DKTK), Heidelberg, Germany.

*These authors contributed equally.

${ }^{\dagger}$ Equal last authors.
}

Submitted July 8, 2017; accepted for publication January 29, 2018.

The authors have disclosed that they have no financial interests, analyses; and further tests dictated by the clinical picture of metastasis. ${ }^{1}$ Recently, clinical studies have used molecular panel sequencing with the hope that a better understanding of the molecular signatures might facilitate the allocation of the respective tumors to the most likely primary. ${ }^{2-5}$ Molecular profiling also offers the pros- arrangements, affiliations, or commercial interests with the manufacturers
of any products discussed in this article or their competitors.
This work was supported by the Volkswagenstiftung (Lichtenberg program
to M.R.S.) and by the German Cancer Consortium (DKTK; to P.S. and A.S.).
Correspondence: Alwin Krämer, MD, Clinical Cooperation Unit Molecular
Hematology/Oncology, German Cancer Research Center (DKFZ), and
Department of Internal Medicine V, University of Heidelberg, Im
Neuenheimer Feld 280, 69120 Heidelberg, Germany.
Email: a.kraemer@dkfz.de; and
Albrecht Stenzinger, MD, Institute of Pathology, University Hospital
Heidelberg, Im Neuenheimer Feld 224, 69120 Heidelberg, Germany.
Email: albrecht.stenzinger@med.uni-heidelberg.de 
Bochtler et al

pect and holds the promise of identifying mutations targetable by molecular therapies critically needed in this entity, due to its generally disappointing response to standard chemotherapy and overall dismal prognosis. Our center has implemented a strategy of performing routine molecular panel sequencing for patients with CUP. In patients with an antecedent or concurrent malignancy in which clonal relation to the CUP tumor is questionable, we have adopted a strategy for panel sequencing of both tumor specimens for comparison to address their clonal relationship.

\section{Case Report}

\section{Clinical Case Description}

This case report presents a male patient with BAP1 (BRCA1-associated protein-1) tumor predisposition syndrome (TPDS) who developed 3 different malignancies.

The first malignancy was a colon cancer in 1984 at 36 years of age, which was treated with right-sided hemicolectomy alone. Twenty-eight years later, the patient was diagnosed with left-sided breast cancer, stage pT1c,pNO (0/5 sn),cM0. Histologic workup revealed a poorly differentiated (G3; ki-67 index, $20 \%$ ) invasive hormone receptor-positive (estrogen receptor [ER], 12/12; progesterone receptor [PR], 8/12; HER2/neu, 1+) ductal adenocarcinoma of luminal type $B$, which was treated with surgical resection and subsequent adjuvant tamoxifen, which is still ongoing at time of writing.

In July 2016, a suspicious mass in the lower right lung together with enlarged mediastinal lymph nodes prompted video-assisted thoracoscopic surgery (VATS). During this procedure, retrosternal lymphadenectomy, thymectomy, and partial resection of the right lower lung were performed. External histologic workup identified the lung lesion as chondroid hamartoma, whereas the lymph nodes were diagnosed with infiltrates of a poorly differentiated adenocarcinoma without traces of the tissue of origin. Given that the immunohistologic profile was clearly divergent from that of the breast cancer, the tumor infiltrates in the lymph node were regarded as yet another distinct tumor type. Accordingly, the clinical diagnosis of CUP was made.

Subsequently, the patient presented to our center, and we performed targeted deep next-generation sequencing (NGS) using formalin-fixed, paraffinembedded tumor tissue of the breast cancer and the CUP to clarify the clonal origin of both tumors. Both malignancies harbored a BAP1 mutation, which we interpreted either as proof of common clonal origin or as an indication of an underlying predisposing germline mutation. The latter hypothesis was also supported by the allelic frequency of the mutation. In retrospect, the enlarged mediastinal lymph nodes could be traced back to CT scans from 2012, suggesting slow clinical progression.

A postoperative PET/CT scan in August 2016 showed persisting suspicious right-sided prepericardial and left-sided paracardial lymph nodes, and therefore VATS was again performed in January 2017 along with decortication, mediastinal lymph node resection, and partial pericardial resection. This time, meticulous histologic and immunohistochemical workup led to the diagnosis of epithelioid pleural mesothelioma, although there was no history of asbestos exposure. Because genetic testing of peripheral blood DNA confirmed the germline nature of the BAP1 mutation, the diagnosis of epithelioid mesothelioma caused by the BAP1-TPDS was established.

With the knowledge of the histologic findings and the BAP1 mutation now at hand, a retroumbilical mass already deemed suspicious on the PET/CT in August 2016 was biopsied during diagnostic laparoscopy in March 2017, and confirmed to be a peritoneal manifestation of epithelioid mesothelioma. Given the typically more benign course of BAP1associated mesotheliomas, we and the patient have been hesitant to start further treatment and have followed a watch-and-wait strategy instead. As of December 2017, the patient had not required therapy.

\section{Histologic and Immunohistologic Findings}

Histologic workup from surgery specimens in January 2017 at our center showed epithelioid neoplasia in tune with epithelioid mesothelioma. Immunohistochemistry (all stains were performed with a BenchMark ULTRA automated slide stainer [Ventana Medical Systems, Inc.]) was positive for the pan-cytokeratin markers AE1/3, CK 5/6, CK7, D2-40, calretinin, WT1, and thrombomodulin, and negative for BerEp4, chromogranin A, TTF1, napsin, CDX2, CK20, HMB45, melan A, CD34, CD45R (LCA), prostate-specific antigen, and androgen receptor (Figure 1A-H). For BAP1 immunohistochemistry, a 
mouse monoclonal antibody (clone C-4) against amino acids 430 to 729 (Santa Cruz Biotechnology Inc.) showed loss of expression in all tumor cells, whereas intermingled lymphocytes displayed retained expression (Figure 1J, H).

\section{Targeted Sequencing}

Molecular panel sequencing was performed using the Oncomine Comprehensive Assay (Thermo Fisher Scientific), which covers 143 malignancy-related genes, and subsequent NGS with an Ion GeneStudio S5 system (Thermo Fisher Scientific). All 3 specimens (breast cancer, 2012; CUP lymph node, July 2016; epithelioid mesothelioma, January 2017) harbored a c.371C $>\mathrm{T}$ mutation in exon 5 of BAP1 (NM_004656), leading to a p.Pro124Leu amino acid replacement. Allele frequencies ranged from $51.9 \%$ to $73.6 \%$ (Table 1). This type of mutation has not yet been deposited in the respective databanks as pathogenic or a mere polymorphism; however, the affected amino acid is highly conserved and all major in silico analyses predict the BAP1 mutation to be damaging/ deleterious (for a detailed account see Table 2).

The breast tumor specimen harbored an additional private WT1 mutation (p.Asp410Glu) at an allele frequency of $18.2 \%$. This mutation is a presumably deleterious mutation, which was undetectable in the lymph node and mesothelioma specimens.

The patient subsequently received genetic counseling by a human geneticist and underwent germline genetic testing, which confirmed the germline nature of the BAP1 mutation. Markedly, other mutations implicated as major drivers in malignant mesotheliomas such as TP53, NF2, and CDKN2A ${ }^{6}$ were included within our molecular panel but could not be detected in any of the 3 samples from our patient.

Using an in-house-developed homologous repair deficiency panel that covers 35 core genes (among them ATM, ATR, CHEK1/2, MSH2/6, RAD50, $R A D 51 C / D)$, further genetic analysis revealed missense mutations of ERCC5 (p.Arg263Gln; 47.7\% COSM4665779) and RECQL4 (p.Ser445Thr; 49\%) (Table 2).

\section{Family History}

The patient's family history revealed a cancer predisposition on the father's side in the ancestral tree, with the father himself, 1 aunt, 1 brother, 3 first cousins, and 1 niece having malignancies, including
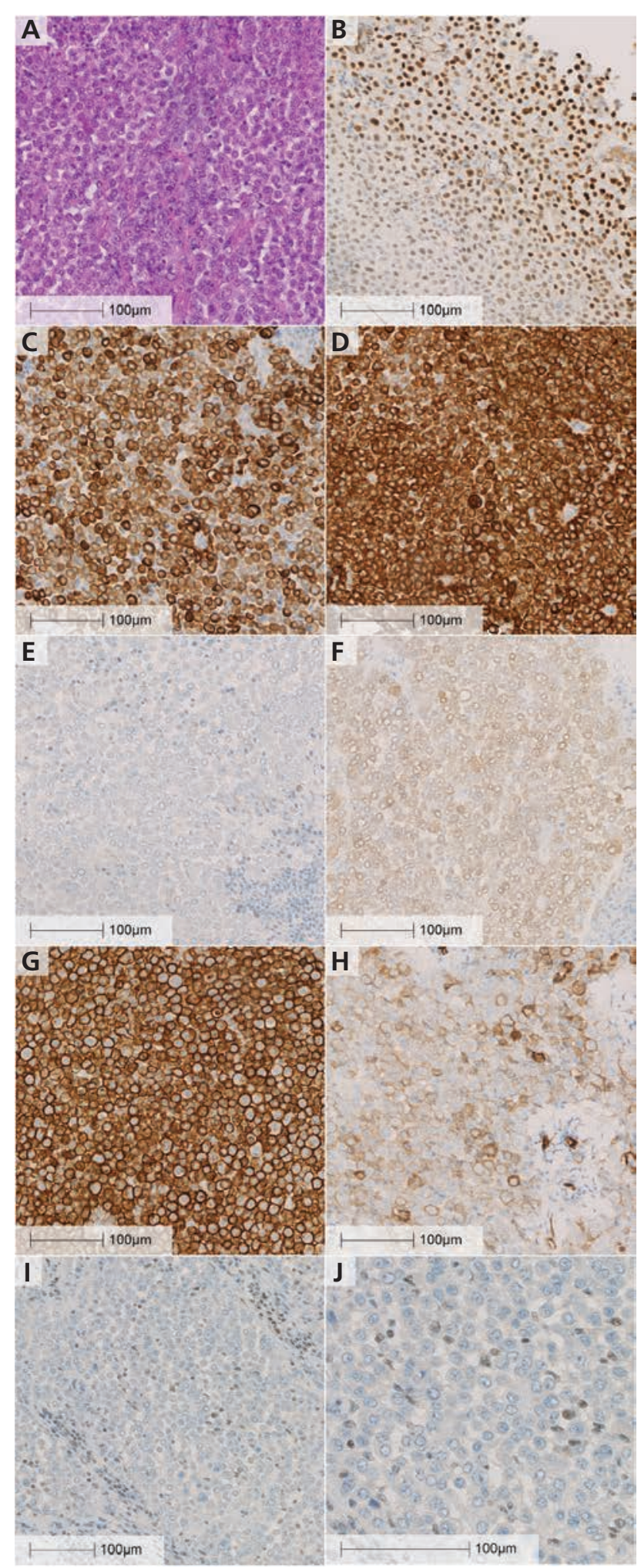

Figure 1. Immunohistochemistry of presumed cancer of unknown primary specimen, allowing the allocation to epithelioid mesothelioma and revision of diagnosis: (A) hematoxylin-eosin stain, (B) WT1, (C) CK7, (D) CK5/6, (E) Ber-EP4, (F) calretinin, (G) D2-40, (H) thrombomodulin, and $(I, J)$ BAP1. Original magnifications: $(A-I) \times 20,(J) \times 40$. 
Bochtler et al

\begin{tabular}{|lcccc|}
\hline \multicolumn{4}{|c|}{ Table 1. Results of Molecular Panel Sequencing } \\
\hline & $\begin{array}{c}\text { Tumor Purity } \\
\text { of Specimen }\end{array}$ & Gene & Mutation & $\begin{array}{c}\text { Allele } \\
\text { Frequency }\end{array}$ \\
\hline $\begin{array}{l}\text { Breast cancer } \\
\text { (Dx: 2012) }\end{array}$ & $70 \%$ & BAP1 & p.Pro124Leu & $65.5 \%$ \\
\hline $\begin{array}{l}\text { Lymph node } \\
\text { CUP/epithelioid } \\
\text { mesothelioma } \\
\text { in retrospect } \\
\text { (Dx: 7/2016) }\end{array}$ & $65 \%$ & BAP1 & p.Pro124Leu & $51.9 \%$ \\
$\begin{array}{l}\text { Epithelioid } \\
\text { mesothelioma } \\
\text { (Dx: 1/2017) }\end{array}$ & $80 \%$ & BAP1 & p.Pro124Leu & $73.6 \%$ \\
\hline
\end{tabular}

Abbreviations: CUP, cancer of unknown primary; Dx, diagnosis.

2 cases of glioblastoma and 1 case each of gastric, liver, and breast cancers and Hodgkin lymphoma. A first cousin also had uveal melanoma and colorectal cancer. The cases of breast cancer and Hodgkin lymphoma emerged in the patient's 40s, whereas all other tumors were diagnosed at age $\geq 70$ years (for a detailed account see Figure 2).

\section{Discussion}

This case highlights the value of integrated histologic and molecular analysis in deciphering some cases of CUP. In this particular case, the correct diagnosis of epithelioid mesothelioma had been missed by a primary and a reference pathologist after initial thoracoscopy, and was only tentatively made after a second thoracoscopy. Knowledge of the BAP1 mutation was crucial to establish and confirm the diagnosis of epithelioid mesothelioma. At our center, we have established a targeted deep NGS program for all patients with CUP. To this end, we are using the Oncomine Comprehensive Assay that is also used in the NCI$\mathrm{MATCH}$ trial, $^{7}$ as well as a validated laboratory- developed test covering 35 genes involved in DNA repair. When metastases in different organs are only ambiguously related, or when patients have previously had a primary malignancy that cannot be safely ruled out as the origin of the CUP, sequencing of all respective samples is performed. In this case, this approach aimed to establish clonal relationships and to gain clues regarding the elusive primary, warranted by the suspicion of an underlying germline mutation.

BAP1 is a deubiquitylase that impacts cell cycle progression, differentiation, and cell death. ${ }^{8}$ Germline mutations of BAP1 have been shown to confer a hereditary familial cancer syndrome called BAP1TPDS (Mendelian Inheritance in Man Tumor Predisposition Syndrome \#614327).9,10 This syndrome is inherited in an autosomal-dominant manner wherein one allele of the tumor suppressor BAP1 is abrogated through a germline mutation, eventually followed by a secondary somatic hit that targets the second allele. ${ }^{11,12}$ The BAP1 mutation in our case was not a truncating but a missense mutation, which is in line with previous reports. ${ }^{12,13}$ In keeping with these data, we observed a selective loss of BAP1 expression in the tumor cells that may be explained by somatic ploidy including loss of the wild-type allele. Coverage data support this hypothesis. Other causes could be methylation of the wild-type allele or abrogative mutations in the promoter- or UTR region of BAP1, which are not covered by the gene panel used here. In any case, together with previous reports, ${ }^{14}$ our results suggest that BAP1 immunohistochemistry can serve as a screening tool for the identification of BAP1-mutated cancers in routine histology diagnostics. Further mutations could be detected in ERCC5

\section{Table 2. In Silico Prediction Analysis}

\begin{tabular}{|c|c|c|c|c|c|c|c|c|c|c|c|c|c|c|c|c|}
\hline \multirow[b]{3}{*}{$\begin{array}{l}\text { Chromosomal } \\
\text { Location }\end{array}$} & \multirow[b]{3}{*}{ Gene } & \multirow[b]{3}{*}{ Exon } & \multirow[b]{3}{*}{$\begin{array}{l}\text { Coding DNA } \\
\text { Exchange }\end{array}$} & \multirow{3}{*}{$\begin{array}{l}\text { Reference } \\
\text { Sequence ID }\end{array}$} & \multicolumn{6}{|c|}{ PolyPhen } & & & & & & \\
\hline & & & & & \multicolumn{2}{|c|}{ SIFT $^{a}$} & \multicolumn{2}{|c|}{ HDIV $^{b}$} & \multicolumn{2}{|c|}{ HVAR $^{c}$} & \multicolumn{2}{|c|}{ MutationTaster $^{\mathrm{d}}$} & \multicolumn{2}{|c|}{ MutationAssessor ${ }^{\mathrm{e}}$} & \multicolumn{2}{|c|}{ FATHMM $^{f}$} \\
\hline & & & & & Score & Pred & Score & Pred & Score & Pred & Score & Pred & Score & Pred & Score & Pred \\
\hline $3 p 21.1$ & $B A P 1$ & 5 & c. $371 C>T$ & NM_004656 & 0 & D & 1 & D & 0.999 & D & 1 & D & 3.75 & $\mathrm{H}$ & 0.69 & $\mathrm{~T}$ \\
\hline $8 q 24.3$ & RECQL4 & 7 & c. $1334 \mathrm{G}>\mathrm{C}$ & NM_004260 & 0 & N/A & 0 & N/A & 0 & $\mathrm{~N} / \mathrm{A}$ & 0 & $\mathrm{~N} / \mathrm{A}$ & 0 & N/A & 0 & N/A \\
\hline $13 q 33.1$ & $\begin{array}{l}\text { BIVM- } \\
\text { ERCC5 }\end{array}$ & $\begin{array}{r}7 \\
15\end{array}$ & $\begin{array}{l}\text { c. } 788 \mathrm{G}>\mathrm{A} \\
\text { c. } 2150 \mathrm{G}>\mathrm{A}\end{array}$ & NM_000123 & 1 & $\mathrm{~T}$ & 0.011 & B & 0.002 & B & 1 & D & -1.06 & $\mathrm{~N}$ & 2.23 & $T$ \\
\hline
\end{tabular}

Abbreviations: HDIV, HumDiv (program to identify human damaging mutations by assuming differences between human proteins and their closely related mammalian homologs as nondamaging); HVAR, HumVar (program to identify human disease-causing mutations by assuming common human nsSNPs as nondamaging); N/A, not applicable; pred, prediction; SIFT, Sorting Intolerant From Tolerant (program to predict whether an amino acid substitution affects protein function).

aSIFT (sift): D, deleterious (sift $\leq 0.05$ ); T, tolerated (sift $>0.05$ ).

bPolyPhen 2 HDIV (pp2_hdiv): D, probably damaging ( $\geq 0.957)$; P, possibly damaging $(0.453 \leq p p 2$ hdiv $\leq 0.956)$; B, benign (pp2_hdiv $\leq 0.452$ )

"PolyPhen 2 HVar (pp2_hvar): D, probably damaging ( $\geq 0.909) ;$, possibly damaging $\left(0.447 \leq p p 2 \_h d i v \leq 0.909\right) ; B$, benign (pp2_hdiv $\left.\leq 0.446\right)$.

dMutationTaster (mt): A, "disease_causing_automatic"; D, "disease_causing"; N, "polymorphism"; P, "polymorphism_automatic."

eMutationAssessor (ma): $\mathrm{H}$, high; $\mathrm{M}$, medium; L, low; $\mathrm{N}$, neutral; $\mathrm{H} / \mathrm{M}$ means functional and $\mathrm{L} / \mathrm{N}$ is nonfunctional.

${ }^{\mathrm{f}}$ FATHMM (fathmm): $\mathrm{D}$, deleterious; T, tolerated. 


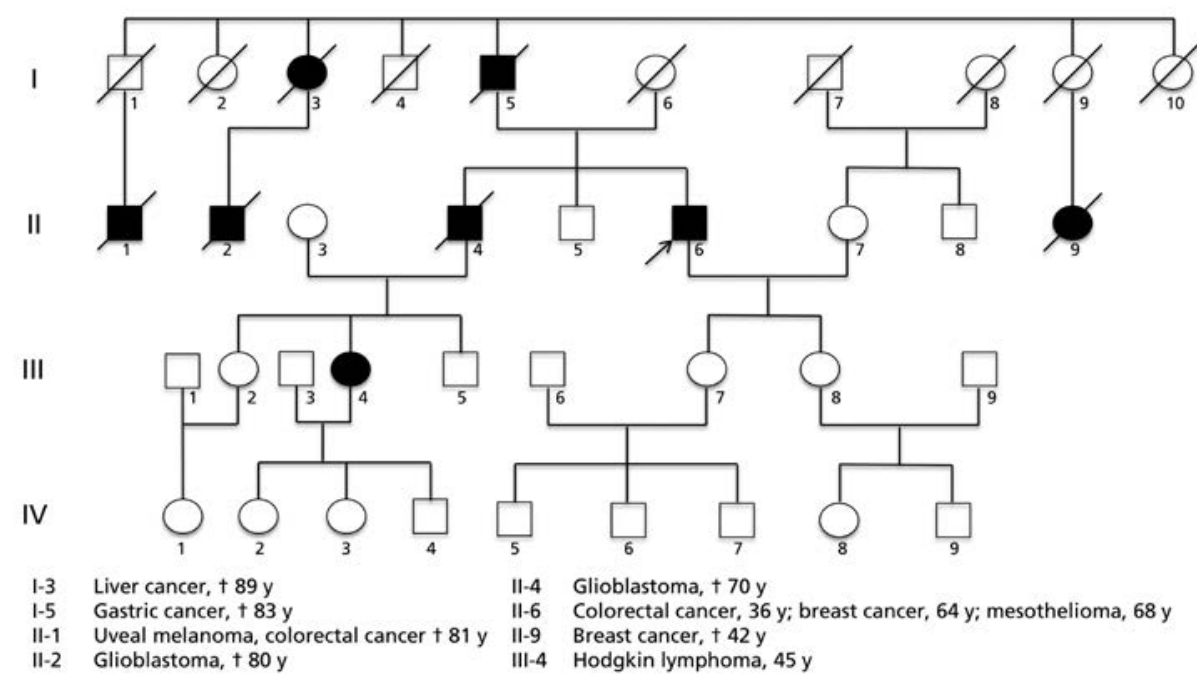

Figure 2. Ancestral tree with clustering of malignancies on the paternal side. Occurrence of malignancy is indicated by black color, and a fatal course by crossed boxes. The index patient reported in this manuscript is marked by an arrow.

'Deceased. The age of death is also indicated.

and RECQL4, which are involved in DNA excision and double-strand break repair. However, the functional role of these mutations remains elusive.

The clinical picture in our case is consistent with previous reports on BAP1-associated tumors with epithelioid mesotheliomas as the characteristically prevailing tumor entity. ${ }^{8,10,15}$ The concurrent manifestation of pleural and peritoneal mesothelioma in our patient has also been reported previously, although most patients display mesothelioma only of either pleura or peritoneum. ${ }^{10}$ Overall, the frequency of BAP1 mutations in mesotheliomas appears to be in the $>20 \%$ range. ${ }^{9,16-18}$ When copy number losses are also considered, the frequency adds up to $>40 \% .{ }^{17,18}$ Cases with epithelioid subtype, familial clustering, or peritoneal involvement appear to display even higher frequencies. ${ }^{19}$

In keeping with the known high frequency of additional malignancies in patients with BAP1TPDS, our patient had colon and breast cancer, a constellation unusual enough in a male patient to raise the suspicion of a predisposing BAP1 germline mutation. ${ }^{10,20,21}$
Our case also recapitulates the known, less aggressive course of BAP1-associated epithelioid mesotheliomas compared with other mesotheliomas. ${ }^{10}$ In our case, the enlarged lymph nodes resected in 2016 were already detectable in 2012, and had only shown slow progression henceforth. Likewise, the peritoneal mesothelioma was visible on a PET/CT performed in 2016, but has not required therapy yet.

Interestingly, one of the patient's first cousins who had colorectal cancer also had uveal melanoma. Given that the latter malignancy is a hallmark of BAP1-TPDS, it strongly implies inheritance, and not de novo mutation in our patient. A possible hereditary predisposition regarding the other malignancies within the family is uncertain given the type of cancers and ages of onset.

The diagnosis of BAP1 TPDS brings the advantage of offering affected individuals timely screening for cancers frequently associated with this entity, such as uveal and cutaneous melanomas. ${ }^{21}$ Promising targeted therapies for BAP1-mutated tumors are being developed, potentially broadening the therapeutic spectrum in the near future. . $2,23^{2}$

\section{References}

1. Varadhachary GR, Raber MN. Carcinoma of unknown primary site. $N$ Engl J Med 2014;371:2040.

2. Tothill RW, Li J, Mileshkin L, et al. Massively-parallel sequencing assists the diagnosis and guided treatment of cancers of unknown primary. J Pathol 2013;231:413-423.
3. Gatalica Z, Millis SZ, Vranic S, et al. Comprehensive tumor profiling identifies numerous biomarkers of drug response in cancers of unknown primary site: analysis of 1806 cases. Oncotarget 2014;5:12440-12447.

4. Ross JS, Wang K, Gay L, et al. Comprehensive genomic profiling of carcinoma of unknown primary site: new routes to targeted therapies. JAMA Oncol 2015;1:40-49. 
Bochtler et al

5. Loffler H, Pfarr N, Kriegsmann M, et al. Molecular driver alterations and their clinical relevance in cancer of unknown primary site. Oncotarget 2016; 7:44322-44329.

6. Guo G, Chmielecki J, Goparaju C, et al. Whole-exome sequencing reveals frequent genetic alterations in BAP1, NF2, CDKN2A, and CUL1 in malignant pleural mesothelioma. Cancer Res 2015;75:264-269.

7. Lih CJ, Harrington RD, Sims DJ, et al. Analytical validation of the next-generation sequencing assay for a nationwide signal-finding clinical trial: Molecular Analysis for Therapy Choice clinical trial. J Mol Diagn 2017;19:313-327.

8. Carbone M, Yang H, Pass HI, et al. BAP1 and cancer. Nat Rev Cancer 2013;13:153-159.

9. Testa JR, Cheung M, Pei J, et al. Germline BAP1 mutations predispose to malignant mesothelioma. Nat Genet 2011;43:1022-1025.

10. Baumann F, Flores E, Napolitano A, et al. Mesothelioma patients with germline BAP1 mutations have 7-fold improved long-term survival. Carcinogenesis 2015;36:76-81.

11. Kadariya $Y$, Cheung $M, X u$ J, et al. Bap1 is a bona fide tumor suppressor: genetic evidence from mouse models carrying heterozygous germline Bap1 mutations. Cancer Res 2016;76:2836-2844.

12. O'Shea SJ, Robles-Espinoza CD, McLellan L, et al. A population-based analysis of germline BAP1 mutations in melanoma. Hum Mol Genet 2017;26:717-728.

13. Leblay N, Leprêtre F, Le Stang N, et al. BAP1 is altered by copy number loss, mutation, and/or loss of protein expression in more than $70 \%$ of malignant peritoneal mesotheliomas. J Thorac Oncol 2017;12:724-733.
14. Shah AA, Bourne TD, Murali R. BAP1 protein loss by immunohistochemistry: a potentially useful tool for prognostic prediction in patients with uveal melanoma. Pathology 2013;45:651-656.

15. Cheung M, Talarchek J, Schindeler K, et al. Further evidence for germline BAP1 mutations predisposing to melanoma and malignant mesothelioma. Cancer Genet 2013;206:206-210

16. Zauderer MG, Bott M, McMillan R, et al. Clinical characteristics of patients with malignant pleural mesothelioma harboring somatic BAP1 mutations. J Thorac Oncol 2013;8:1430-1433.

17. Bott M, Brevet M, Taylor BS, et al. The nuclear deubiquitinase BAP1 is commonly inactivated by somatic mutations and $3 \mathrm{p} 21.1$ losses in malignant pleural mesothelioma. Nat Genet 2011;43:668-672.

18. Yoshikawa $Y$, Emi M, Hashimoto-Tamaoki $T$, et al. High-density arrayCGH with targeted NGS unmask multiple noncontiguous minute deletions on chromosome 3p21 in mesothelioma. Proc Natl Acad Sci U S A 2016;113:13432-13437.

19. Alakus H, Yost SE, Woo B, et al. BAP1 mutation is a frequent somatic event in peritoneal malignant mesothelioma. J Transl Med 2015;13:122.

20. Rai K, Pilarski R, Cebulla CM, Abdel-Rahman MH. Comprehensive review of BAP1 tumor predisposition syndrome with report of two new cases. Clin Genet 2016;89:285-294.

21. Pilarski R, Cebulla CM, Massengill JB, et al. Expanding the clinical phenotype of hereditary BAP1 cancer predisposition syndrome, reporting three new cases. Genes Chromosomes Cancer 2014;53:177-182.

22. Schunselaar LM, Zwart W, Baas P. Targeting BAP1: a new paradigm for mesothelioma. Lung Cancer 2017;109:145-146.

23. LaFave LM, Beguelin W, Koche R, et al. Loss of BAP1 function leads to EZH2-dependent transformation. Nat Med 2015;21:1344-1349.

\section{Recorded Presentations}

\section{ONLINE NOW}

\section{NCCN 23rd Annual Conference:}

\section{Improving the Quality, Effectiveness, \& Efficiency of Cancer Care ${ }^{T M}$}

\section{education.nccn.org/ac2018}

These activities are approved for AMA PRA Category 1 Credit ${ }^{\mathrm{TM}}$ for physicians and will award contact hours for nurses, pharmacists, and other health care professionals. Complete accreditation information is available online.

These activities are supported by educational grants from: Astellas; AstraZeneca; Biocompatibles, Inc. (BTG); Celgene Corporation; Genentech; Janssen Biotech, Inc., administered by Janssen Scientific Affairs, LLC.; Jazz Pharmaceuticals, Inc. Lilly; Novartis; Pfizer; Prometheus Laboratories Inc.; Sanofi Genzyme; and Taiho Oncology. These activities are supported by independent educational grants from: AbbVie; Boehringer Ingelheim Pharmaceuticals, Inc.; Incyte; and Merck \& Co., Inc. These activities are supported by an unrestricted educational grant from Gilead Sciences, Medical Affairs. These activities are supported by educational funding provided by Amgen. 